\title{
Comentario editorial: Dejar de fumar durante el embarazo: cómo ayudar a las embarazadas trabajando con los proveedores de salud
}

\author{
Quiting smoking during pregnancy: how to help pregnant woman working with health providers
}

Mabel Berrueta *

El tabaquismo es la primera causa de muerte evitable en el mundo. En Argentina, el consumo de tabaco causa la muerte de más de 40.000 personas por año. Tres de cada diez adultos son fumadores y, de acuerdo con diversas encuestas realizadas entre 2001 y $2009^{1-4}$, Argentina es uno de los que tiene mayor proporción de mujeres fumadoras (con un rango que va de 24 a $33 \%$ en distintas edades y subgrupos).

¿Qué sucede con esas mujeres cuando están embarazadas? Según una encuesta que se realizó durante controles prenatales en Argentina y Uruguay ${ }^{5}, 30 \%$ deja de fumar antes de embarazarse o en el momento en que se entera que está embarazada; el $5 \%$ por ciento deja en el transcurso del embarazo y entre 10 y $20 \%$ de ellas continúa fumando.

El consumo de tabaco durante el embarazo tiene claros y evidentes peligros para la mujer, el feto y el recién nacido. Es mayor el riesgo de sufrir abortos, embarazos ectópicos, desprendimientos de placenta y partos prematuros, además de la probabilidad de que el recién nacido tenga bajo peso, malformaciones congénitas y sufra muerte súbita del lactante.

Las mujeres embarazadas constituyen entonces una particular subpoblación blanco de intervenciones para dejar de fumar durante el embarazo y para mantener ese cambio de conducta (especialmente porque de las mujeres que logran abandonar su adicción durante el embarazo, el 50\% vuelve a fumar dentro de los seis meses después del parto ${ }^{6}$ ).

En este sentido, existe una intervención breve, también llamada las "5A" (por los conceptos Averiguar, Aconsejar, Asistir, Ayudar y Acompañar) que, acompañada de materiales de autoayuda específicos, aumenta la cesación tabáquica entre las embarazadas fumadoras y constituye el estándar de tratamiento a nivel internacional.

La Guía Nacional de Tratamiento de la Adicción al Tabaco de 2011 del Ministerio de Salud de la Nación sugiere que todos los equipos de salud deben estar capacitados para realizar las " $5 A^{\prime}$ " en todos los pacientes. Sin embargo, esta intervención sigue siendo subutilizada por los hospitales y centros de atención primaria de nuestros países. Por ejemplo, según datos provenientes de 20 hospitales en Argentina y Uruguay, menos del $5 \%$ de las mujeres fumadoras están recibiendo ésta intervención breve en todas las visitas prenatales.

Quizás uno de los temas más críticos que impide mejorar la salud pública de la población radica en el enorme abismo que existe entre lo que sabemos que puede optimizar la salud y su cuidado, y lo que en realidad se usa e implementa cada día en la práctica médica.

Reproducido por cortesía del Instituto de Efectividad Clínica y Sanitaria: http://www.iecs.org.ar/iecs-visor-newsletter1.php?cod_producto=755\&origen=publicacion

Para reducir esta brecha, un grupo de investigadores del Instituto de Efectividad Clínica y Sanitaria (IECS) en colaboración con el Programa Nacional de Control de Tabaco y junto a investigadores del Centro de Control de Enfermedades de los EE.UU. (en inglés: CDC), está conduciendo una investigación en 20 hospitales y Centros de Atención Primaria del sector público que atienden control prenatal en Argentina y Uruguay. Su objetivo primario es evaluar una intervención multifacética destinada a incrementar la frecuencia de mujeres que reciban las " $5 \mathrm{~A}$ " durante el control prenatal.

Esta intervención incluye la diseminación de estas recomendaciones y la sensibilización de los proveedores de salud (médicos, obstétricas y enfermeras) en este problema, una capacitación en la intervención breve de las " 5 A" y un soporte para implementar la consejería en el ámbito del control prenatal. Esta intervención fue diseñada sobre la base de una investigación cualitativa realizada previamente en ambos países y a los efectos de este estudio, con el objetivo de poder contar con información local sobre las barreras y los facilitadores para la implementación de intervenciones para la cesación tabáquica durante el control prenatal.

Así es como hoy sabemos que los proveedores de salud dicen no tener conocimiento adecuado sobre estas intervenciones, no sentirse competentes para ayudar a una embarazada a dejar de fumar y estar poco confiados en la utilidad de las mismas durante el control prenatal. También opinan que hay algunos inconvenientes en el sistema de salud y sugieren como principal barrera la falta de tiempo, de recursos, de comunicación entre pares y con superiores, y de políticas prioritarias sobre tabaquismo en las instituciones.

Por su parte, las mujeres fumadoras embarazadas manifiestan tener poco conocimiento de los riesgos reales del cigarrillo durante esta etapa de sus vidas y sobre la existencia de una consejería para dejar de fumar. Además, subestiman la potencial ayuda del profesional a cargo del control prenatal para que puedan abandonar el cigarrillo.

Como principales facilitadores se identificaron la predisposición al cambio de los proveedores sanitarios, la necesidad de utilizar tiempos muertos (como los de la sala de espera en hospitales) y la manifiesta necesidad de las embarazadas de tener más y mejor comunicación con los proveedores de salud y de recibir conocimiento y material impreso sobre el problema.

La meta que persigue el IECS es diseñar intervenciones para incrementar el uso de prácticas probadas como beneficiosas para el cuidado de la salud y evaluarlas en el contexto de ensayos clínicos rigurosos, con el fin de traducir a la práctica diaria los principales hallazgos de la ciencia.

Recibido el 15/08/2012 y aceptado el 03/09/12

Referencias

1. Global Youth Tobacco Survey (GYTS). Differences in Worldwide Tobacco Use by Gender: Findings from the Global Youth Tobacco Survey. Journal of School Health 2003;73: 6 . 2. Ministerio de Salud y Ambiente de la Nación (MSAN). Encuesta de Tabaquismo en Grandes Ciudades de Argentina. MSAL, Argentina, 2004. En: www.msal.gov.ar/htm/site_tabaco/vigilancia.asp; consultado el 20/06/2012.

3. Míguez, H. Informe de resultados del Global Youth Tobacco Survey en Buenos Aires, Argentina. Centre for Disease Control, Argentina, 2000. En: http://www.geocities. com/hugomiguez/taba.html; consultado el 20/06/2012.

4. Segunda Encuesta Nacional de Factores de Riesgo para Enfermedades No Transmisibles., Primera Edición. Buenos Aires. Ministerio de Salud de la Nación, 2011

4. Segunda Encuesta Nacional de Factores de Riesgo para Enfermedades No Transmisibles., Primera Edición. Buenos Aires. Ministerio de Salud de la Nación, 2011
5. Althabe F., Colomar M, Gibbons L, Belizan J, Buekens P., Tabaquismo durante el embarazo en Argentina y Uruguay - Medicina (B. Aires) v.68 n.1 -ene./feb. 2008

6. 2008 Pregnancy Risk Assessment and Monitoring System (PRAMS) http://www.cdc.gov/prams/; consultado 20/06/2012

* Departamento de Investigación en Salud de la Madre y el Niño del Instituto de Efectividad Clínica y Sanitaria (IECS). 